\title{
In-silico STUDIES OF AMENTOFLAVONE AND ITS DERIVATIVES AGAINST SARS-COV-2
}

\author{
Faisal Maulana Ibrahim ${ }^{1}$, Holis Abdul Holik ${ }^{1, 凶}$ and Arifudin Achmad ${ }^{2}$ \\ ${ }^{1}$ Department of Pharmaceutical Analysis and Medicinal Chemistry, Faculty of \\ Pharmacy/Universitas Padjadjaran, Sumedang- 45363, (West Java) Indonesia \\ ${ }^{2}$ Department of Nuclear Medicine, Faculty of Medicine/Universitas Padjadjaran, Sumedang- \\ 45363, (West Java) Indonesia \\ ${ }^{\circledR}$ Corresponding Author: holis@unpad.ac.id
}

ABSTRACT

The COVID-19 pandemic caused by SARS-CoV-2 infection is of global concern. The SARS-CoV-2 main protease (M ${ }^{\text {pro2 }}$, PDB ID: 6LU7), is an important SARS-CoV-2 enzyme in mediating viral replication and transcription, making it very strategic to be a drug target. Amentoflavone and its derivatives underwent molecular docking and 3D liganbased pharmacophore modeling to analyze their molecular interactions against $\mathrm{M}^{\text {pro2 }}$ and evaluation for their absorption, distribution, metabolism, excretion, (ADME) properties. Fifty-one compounds had better docking parameters than comparison compound (remdesivir) and S29 is the best-docked compound $(\Delta \mathrm{G}=-13.06 \mathrm{kcal} / \mathrm{mol})$. Amentoflavone derivatives are promising candidates for novel anti-COVID agents.

Keywords: COVID-19, SARS-CoV-2, Amentoflavone, Molecular Docking, In-silico

RASĀYAN J. Chem., Vol. 14, No.3, 2021

\section{INTRODUCTION}

Coronavirus disease 2019 (COVID-19) is a contagious and multi-organ disease caused by a viral infection called severe acute respiratory syndrome coronavirus-2 (SARS-CoV-2). SARS-CoV-2 is mainly transmitted through air droplets and direct contact. The disease was first identified in December 2019 in Wuhan, China, and has since spread globally, resulting in the ongoing COVID-19 pandemic. At the end of April 2021, there have been 140 million people confirmed positive for SARS-CoV-2 infection with a total more than 3 million of death. ${ }^{1}$ Vaccine are currently being developed, however; no single antiviral drug is proven effective for severe cases. Discovering antiviral drugs is essential to contain this global burden., ${ }^{2,3}$ SARS-CoV-2, a member of the $\beta$-coronavirus family, is characterized as a single-stranded RNA enveloped in $60-100 \mathrm{~nm}$ diameter oval/round-shaped membrane covered by spike glycoproteins. The SARS-CoV-2 RNA chain ( $\sim 30,000$ nucleotides) has approximately $80 \%$ identical nucleotides sequence to that of SARS$\mathrm{CoV}$, the culprit of the SARS 2003 outbreak. ${ }^{4,5}$ It is comprised of a replicase enzyme gene that encodes two overlapping polyproteins, ppla and pplab, essential for viral replication and transcription. SARS-CoV2 infects cells via membrane fusion initiated by binding between its spike glycoproteins to angiotensinconverting enzyme 2 (ACE2) protein - which is highly concentrated in the surface of the airway epithelial cells. Once viral RNA materials are released into cell cytoplasm and being translated to polyproteins, SARS-CoV-2 main protease $\mathrm{M}^{\text {pro2 }}$ (PDB ID: 6LU7) (also named as chymotrypsin-like protease (3CL ${ }^{\text {pro2}}$ ) leads proteolytic processing by digesting the polyprotein conserved sites, starting with the autolytic cleavage of this enzyme itself from ppla and pplab. This proteolysis generates functional polypeptides to facilitate virus replication (Fig.-1). ${ }^{4}$

The complete structure of SARS-CoV-2 $\mathrm{M}^{\text {pro2 }}$ was discovered in February 2020, two months after the initial outbreak. The SARS-COV-2 $\mathrm{M}^{\text {pro2 }}$ (also named 3CL pro2, Protein Data Bank (PDB) ID: 6LU7) has a remarkable $96 \%$ amino acid sequence identity of SARS-CoV $\mathrm{M}^{\text {pro }}$ (3CL ${ }^{\text {pro }}$, PDB ID: 1UJ1) and their crystal structure is also highly similar. ${ }^{11}$ The catalytic amino acid of $\mathrm{M}^{\text {pro2 }}$ is represented by dyad His 41 and Cys 145 which are conserved in both SARS-CoV and SARS-CoV-2 by cleaving the viral polyprotein at eleven conserved sites through recognizing sequence Leu-Gln $\downarrow$ Ser/Ala/Gly. ${ }^{6}$ The root-mean-square deviation 
RASĀYAN J. Chem.

Vol. 14 | No. 3 |1469-1481| July - September | 2021

(RMSD) of the backbone root between these two proteins is only $1.4 \AA$, less than the $2.0 \AA$ cutoff. $^{7}$ The similarities of their catalytic amino acids and their amino acid target sequence on viral polyprotein are an indicator of efficient inhibition by the same inhibitor.

As a 33.8-kDa enzyme, $\mathrm{M}^{\text {pro2 }}$ is among the highly conserved non-structural SARS-CoV-2 proteins and also lacks closely related homologs in humans. Consequently, inhibiting SARS-CoV-2 $\mathrm{M}^{\mathrm{pro} 2}$ should not be toxic to humans. Since $\mathrm{M}^{\text {pro2 }}$ is indispensable for viral replication and infection processes, inhibiting $\mathrm{M}^{\text {pro2 }}$ enzymatic activity would block the viral life cycle. These properties make $\mathrm{M}^{\text {pro }}$ a valid and very attractive drug target. ${ }^{5,6,8}$
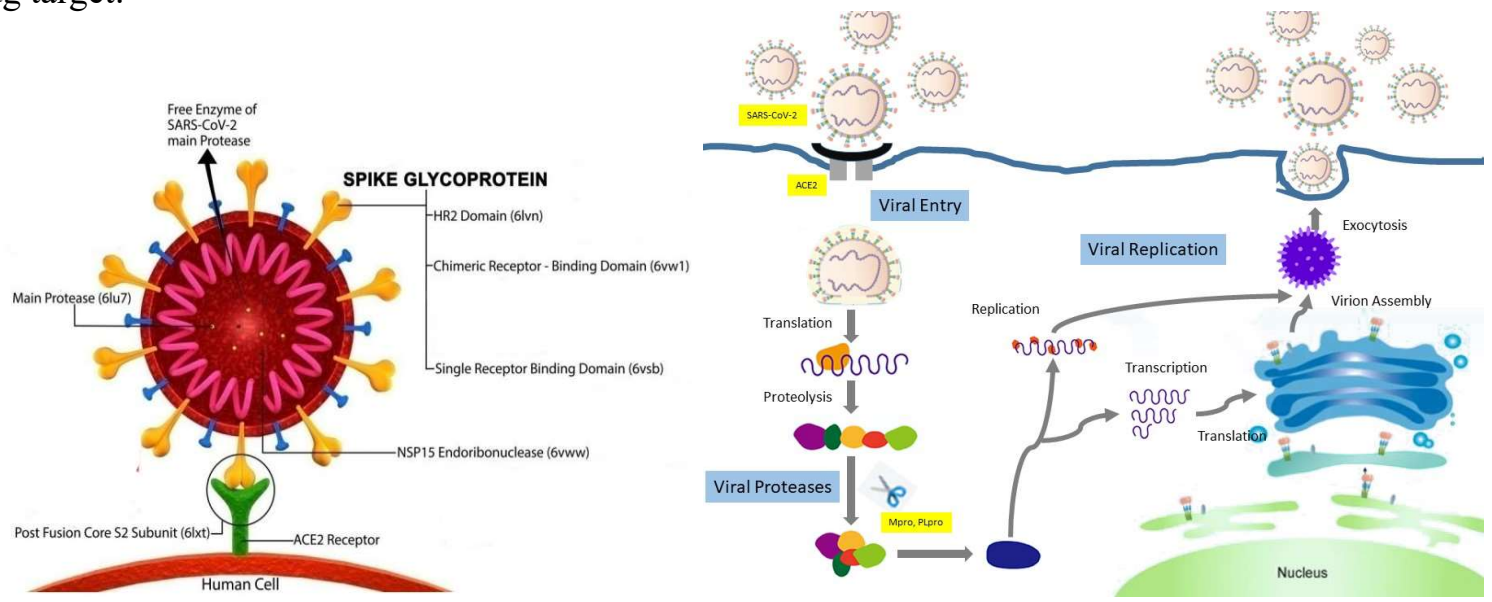

Fig.-1: The Structure of SARS-CoV-2 and its Replication Mechanism

In SARS-CoV-2 infected patients, the immune system responds by mobilizing inflammatory cells. If a bacterial infection concurrent, inflammation might progresses severely and rapidly destroy lung tissue, leads to systemic hypoxia and multi-organ failure which often fatal. ${ }^{9}$ The current COVID-19 treatment guidelines are either loosely based on the regimen involved in The Solidarity Trials or according to local/national guidelines. Drugs evaluated in The Solidarity Trials are anti-Ebola (remdesivir), anti-HIV (lopinavir and ritonavir combined), and a combination of anti-HIV with interferon-beta, each in comparison to the local standard of care. ${ }^{10}$ Generally, antiviral drugs have mild adverse effects, however, their efficacy to disturb SARS-CoV-2 replication is still unclear. Drugs proposed to inhibit viral entry, e.g. camostat mesylate, are still under investigation. ${ }^{11}$ Chloroquine (and hydroxychloroquine) which was presumed able to disturb viral endosomes, is also banned from further research due to its null benefit for COVID-19 patients. ${ }^{10}$ Based on recent reports, there is no anti-SARS-CoV-2 drug with a clear action mechanism that can be relied upon. ${ }^{12}$

Amentoflavone $\left(\mathrm{C}_{30} \mathrm{H}_{18} \mathrm{O}_{10}\right)$, a natural polyphenolic compound found in Calophyllaceae, Clusiaceae, Cupressaceae, Euphorbiaceae, and Selaginellaceae family and Calophyllum, Garcinia, and Selaginella genus is a biflavonoids (dimeric apigenin) which is linked by covalent bonds between C3'-C8' ' atoms (Fig.2).

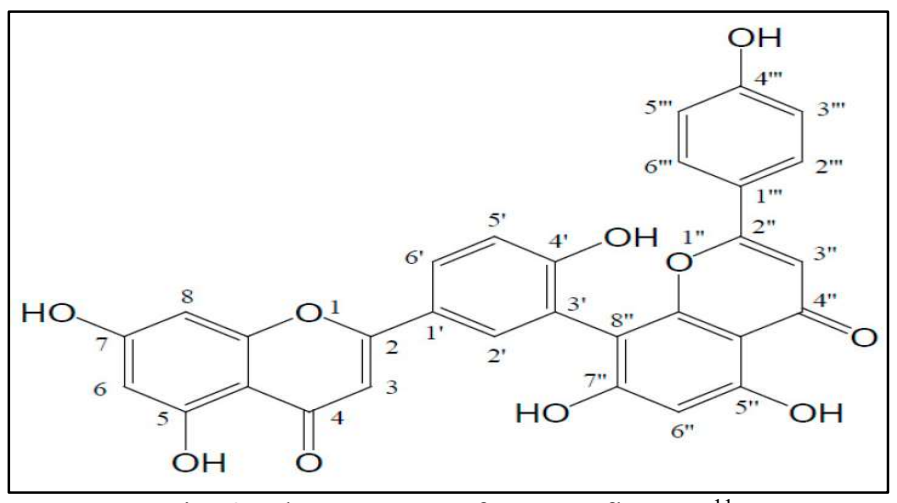

Fig.-2: The Structure of Amentoflavone. ${ }^{11}$ 
RASĀYAN J. Chem.

Vol. 14 | No. 3 |1469-1481| July - September | 2021

The hydroxyl groups on the C5, C7, C4', C5', C7', and C4','atoms can be easily substituted with other groups to obtain derivative compounds. Amentoflavone has many natural derivatives and is also reported to have antiviral and antibacterial activity, including a potent noncompetitive inhibitory activity against $3 \mathrm{CL}^{\text {pro }}$ of SARS-CoV. ${ }^{13,14} \mathrm{M}^{\text {pro2 }}$ also has a similar characteristic as observed in other coronavirus proteases in cleaving proteins after the Gln residue. Hence, amentoflavone and its derivatives might also have inhibitory activity towards $\mathrm{M}^{\text {pro2 }}$. This study reports molecular docking to obtain structural information among amentoflavone and its derivatives against $\mathrm{M}^{\mathrm{pro2}}$ and further virtual screen these pharmacophores as the starting point for structure-guided drug discovery. Due to the mutagenic potential of amentoflavone, it is crucial to obtain suitable derivatives that maintain $\mathrm{M}^{\text {pro2 }}$ inhibitory activity but also have excellent pharmacological properties including the lowest toxicity. ${ }^{15}$

\section{EXPERIMENTAL}

\section{Hardware and Software}

A personal computer with 6-cores 12-threads AMD Ryzen 5 2600, $3.4 \mathrm{GHz}$ CPU with Radeon RX 570 GPU and 16 GB RAM was employed and equipped with the following software for In-silico studies:

1. The ChemOffice 2010 and ChemDraw Ultra 12.0 software (PerkinElmer Inc., downloaded from http://www.cambridgesoft.com) for drawing 2D structures and convert them into 3D structures,

2. LigandScout Advanced 4.1 software (Wolber and Inte: Ligand $\mathrm{GmbH}$, downloaded from http://www.inteligand.com/ligandscout/download.shtml) for pharmacophore modeling,

3. AutoDock 4.2.6 and AutoDockTools 1.5.6 programs (The Scripps Research Institute, downloaded from http://www.autodock.scripps.edu) for molecular docking simulations,

4. BIOVIA Discovery Studio 2017 R2 Client (Dassault Systèmes, downloaded from http://www.accelrys.com/) for visualization of PDB complex, the bond between ligands and receptors, geometry optimization, and overlays during the validation process,

5. PreADMET webserver application (Bioinformatics and Molecular Design Research Center; available at https://preadmet.bmdrc.kr/adme/) for ADME properties evaluation, and

6. admetSAR webserver application (Shanghai Key Laboratory of New Drug Design, China; available at http://lmmd.ecust.edu.cn/admetsar2/ also for ADME properties evaluation.

\section{Acquisition of Chemical Structure}

The 3D structure of the $\mathrm{M}^{\text {pro2 }}$ was downloaded from Protein Data Bank (PDB) (http://www.rscb.org, PDB ID: 6LU7). As a result of the X-ray crystallography depiction, $\mathrm{M}^{\mathrm{pro2}}$ (6LU7) was complexed with N3 inhibitor molecules. The separation was performed using the BIOVIA Discovery Studio. The 3D structure of the ligands (amentoflavone and its derivatives) was drawn and has been optimized using ChemOffice 2010, and ChemDraw Ultra 12.0 (PerkinElmer Inc.). Seven commercially available drugs included in The Solidarity Trial and local COVID-19 treatment guidelines (Azithromicyne, Favipiravir, Lopinavir, Nelfinavir, Oseltamivir, Remdesivir, and Ritonavir) were chosen as comparison compounds. Their 3D structure was downloaded from PubChem (https://pubchem.ncbi.nlm.nih.gov/).

\section{Molecular Docking Simulation}

6LU7 and all ligands (including comparison compounds) were prepared using AutoDockTools 1.5.6. and were protonated by adding hydrogen atoms to the structures. As a macromolecule, 6LU7 was added to Kollman charges, and ligands were added to Gasteiger charges. Grid parameter data was based on a grid box consisting of $26 \times 54 \times 32$ points with a spacing of $0.375 \AA$ and adjusted to the active sides of $6 \mathrm{LU} 7$ at $x$ $=-9,732, y=11,403$, and $z=68,925$. Docking parameter data is based on the Lamarckian Genetic Algorithm (LGA) with 100 runs, 150 population size, 2,500,000 energy evaluation, 0.02 gene mutation rate, and 0.8 rates of crossover. Ligands with the lowest bond energy $(\Delta G)$ to $6 \mathrm{LU} 7$ were selected and each interaction with 6LU7 was further characterized (LigandScout Advanced 4.1). Therefore, as mentioned by Murthy and Nasaiah (2019) and Muchtaridi et. al, all the compounds are ranked based on the docking parameter (Gibbs free energy), key amino acid interaction, several clusters, and inhibition constant. ${ }^{31-33}$

\section{D Ligan-based Pharmacophore Modelling}

The 3D ligan-based-pharmacophore modeling is performed on the X-ray crystallography structure of N3inhibited 6LU7 (LigandScout Advanced 4.1). Amentoflavone and its derivatives were screened through the 
RASĀYAN J. Chem.

Vol. 14 | No. 3 |1469-1481| July - September | 2021

model that has already build with the 3D-ligand based pharmacophore modeling and scored that expressed as a percentage of the pharmacophore-fit score.

\begin{abstract}
ADME Prediction
ADME predictions including Human Intestinal Absorption (HIA), Caco-2 cell absorption (oral absorption estimates), plasma-protein binding (PPB) penetration, and blood-brain barrier (BBB) penetration, and significant descriptors of drug-likeness such as mutagenicity, toxicological dosage level for different tissues, and pharmacologically relevant properties of the compounds were predicted using web applications at PreADMET server (https://preadmet.bmdrc.kr/adme/) and admetSAR server (http://lmmd.ecust.edu.cn/admetsar2/).
\end{abstract}

\title{
Receptor Preparation and Validation
}

RESULTS AND DISCUSSION

The X-ray structure of $\mathrm{M}^{\text {pro2 }}$ (complexed with N3 inhibitor, PDB ID: 6LU7) selected for molecular docking studies to amentoflavone and its derivatives was the first PDB published (March 18, 2020) and the only available $\mathrm{M}^{\text {pro2 }}$ structure when our preliminary docking studies were started: (xavlxx: $\mathrm{N}-[(5$-methyl isoxazole-3-yl)carbonyl] alanyl-L-valyl-L-N 1 -((1R,2Z)-4-(benzyloxy)-4-oxo-1-[[(3R)-2oxopyrrolidin-3-yl]methyl]but-2-enyl)-L-leucinamide). The 6LU7 has already specified with the value of the experimental resolution (2.16 $\AA$ ), R-value work (0.202), R-value free (0.235), and R-value observed (0.204). The X-ray structure revealed that N3 formed a covalent bond with Cys 145 and a conventional hydrogen bond with Glu166, Gln189, Thr190, His163, Gly143, And His 164. ${ }^{11}$

Before molecular docking was performed, N3 was extracted from the $\mathrm{M}^{\text {pro2 }}$ structure and re-docked back to $\mathrm{M}^{\text {pro2 }}$ to verify that the molecular docking software can reproduce the agonist conformation of $\mathrm{N} 3$. The result is valid when an RMSD value obtained is $\leq 2.0 \AA$. $^{7}$ The best-docked N3 conformation showed an RMSD of $1.77 \AA$ compared to the original $\mathrm{M}^{\text {pro2 }}$ X-ray conformation. ${ }^{11}$ (Table-S1, Supplementary Data).

\section{Modification of Amentoflavone to Produce its Derivatives}

The design of amentoflavone and its derivatives are shown in Table-S2 (Supplementary Data). Modified structures of amentoflavone are obtained from natural derivative compounds (NDC) that are present in plants (9 compounds) and from artificial modification. NDC structure data was downloaded from PubChem. Artificial modification of amentoflavone was focused at C7, C4', C7', and C4'"' atoms due to its versatility for substitution with methoxy and methylcyclohexane, in particular, to increase their hydrophobicity. ${ }^{13}$

Besides that, the structure modification is aimed to give higher flexibility to the ligands. This way is conducted to influence the binding mode of interaction of the ligand to its receptor. According to Koshland (1958) as cited by Megantara et.al, the receptor will re-shape its conformation as the penetration of ligand occurred to provide accessibility for the ligand-binding. ${ }^{30}$

Forty-two artificial derivative compounds (ADC) were obtained, coded as H1-H6 and S1-S36. The 2D structure of ADC was drawn with ChemDraw Ultra 12.0 and converted to 3D by Chem3D Ultra 12.0. The predominant conformational ligand structures (the correct geometry and conformation) of each ADC have been determined by energy minimization calculations using the Austin Model (AM1) semi-empirical method.

\section{Drug-likeness Evaluation}

The drug-likeness of amentoflavone derivative compound was identified with Lipinski's Rule of Five (RO5) as follows: molecular mass $<500 \mathrm{Da}$, hydrogen bond donors $<5$, hydrogen bond acceptors $<10$, and $\log \mathrm{P}<5$. A compound that fulfills RO5 (no more than one violation) is associated with $90 \%$ of orally active drugs thus has to achieve phase II clinical status, otherwise, there is a high probability of oral activity problem. ${ }^{16}$ Table-S3 (Supplementary Data) shows that 38 amentoflavone derivative compounds pass RO5 criteria, except their molecular weights $(>500 \mathrm{Da})$. However, since $\mathrm{M}^{\mathrm{pro2}}$ is not a viable target for smallmolecule inhibitor $<500 \mathrm{Da}$, the exception in this regard is amenable for SARS-CoV-2 antiviral drug design, as keeping the rest of the RO5 parameter has met. ${ }^{17}$ 
RASĀYAN J. Chem.

Vol. 14 | No. 3 |1469-1481| July - September | 2021

Table-S3 shows that the best-docked amentoflavone derivative compound (S29) has two RO5 violations (i.e. molecular weight $620.2 \mathrm{~g} / \mathrm{mol}$ and $\log P$ 5.26), thus may not be concluded as a druglike compound. However, $\log \mathrm{P}$ of 5.2 might be acceptable because it falls in the percentile of $10-90 \%$ compared to over 1,000 orally administrated FDA-approved drugs. ${ }^{18}$

Fulfilling the RO5, however, is not a guarantee of drug-likeness because RO5 explained no specific chemistry structural features found in drugs or non-drugs. ${ }^{19}$ Therefore, a compound with more than one RO5 violation can be formulated into non-oral preparation, once their parameters and property range differences from oral drugs have been cautiously considered, in this case as an intravenous or inhalation preparation. ${ }^{18}$ Inhalation drugs tend to have a higher polar surface area (PSA) because pulmonary permeability is less sensitive to polar hydrogen-bonding functionality. ${ }^{20}$ However, inhalation preparation might have its relevance since the most affected by SARS-CoV-2 infection is the respiratory system. ${ }^{9}$

\section{Molecular Docking Simulation}

Targeting the key residues (catalytic amino acid) in the active site of $\mathrm{M}^{\text {pro2 }}$ is the main inhibition strategy to inactivate the $\mathrm{M}^{\text {pro2 }}$ proteolytic function. The active site of $\mathrm{M}^{\text {pro2 }}$ consists of Thr24, Thr26, Phe140, Asn142, Gly143, Cys145, His163, His164, and Glu166 residues while Cys 145 plays the role as the catalytic amino acid during proteolysis by cleaving sequence Leu-Gln $\downarrow$ Ser/Ala/Gly. ${ }^{5}$ The hydrogen bond between protein side chains and the drug is fundamental for inhibition and stability, thus play a significant role in drug-receptor interaction. The distance between the hydrogen-bond donor and acceptor (the shorter, the stronger) determine the strength of a hydrogen bond. ${ }^{21}$

As mentioned above, the $\mathrm{M}^{\text {pro2 }}$ structure was obtained in a form of complex inhibition with N3. Table-2 shows that N3 has two short and strong hydrogen bond: Cys145 (1.8 $\AA$ ) and His163 (2.4 $\AA)$; and five

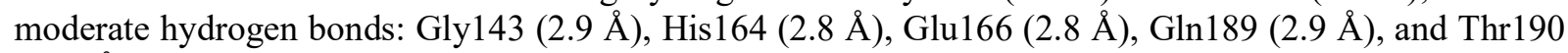
$(2.8 \AA$ ). Table-1 shows molecular docking parameters from seven comparison compounds (drugs commonly used in COVID-19 guideline therapy), lead compound (amentoflavone), NDC, and ADC. Sixtyone compounds had a better affinity $(\Delta \mathrm{G})$ than remdesivir $(-8.38 \mathrm{kcal} / \mathrm{mol})$ and lopinavir $(-9.86 \mathrm{kcal} / \mathrm{mol})$, two promising protease inhibitors involved in The Solidarity Trial. Among amentoflavone derivatives, S29 is the best-docked molecule $(-13.06 \mathrm{kcal} / \mathrm{mol})$ by binding to Glu166, Asn142, Met49, Phe40, Ser144, and more importantly Cys145 residues via hydrogen bonds (Fig.-3), a crucial aspect in $\mathrm{M}^{\text {pro2 }}$ inhibitor design. Besides, the $\mathrm{S} 29-\mathrm{M}^{\text {pro2 }}$ interaction is strengthened and stabilized by several amino acids whose very strong hydrogen bonds: Ser144, His63, Phe140, and Thr190 (Table-1, Fig.-3).

Twenty-four natural and artificial amentoflavone derivatives pass the molecular docking simulation screening based on the following parameter: able to bind Cys145, lowest Gibbs' energy, and highest affinity (smaller Ki) (Table-1, bold): garciniaflavone $\mathrm{A}$, garciniaflavone $\mathrm{F}$, pyranoamentoflavone, taiwanhomoflavone A, taiwanhomoflavone B, H1, H3, H4, H5, S1, S2, S5, S6, S14, S22-24, S26, S27, S29, S30, S32, S34, and S35.

Table-1: Molecular Docking Parameters of Comparison Compound, Amentoflavone, and its Derivatives

\begin{tabular}{c|c|c|c|c|c|c}
\hline \multirow{2}{*}{ No } & Ligand & Origin & $\begin{array}{c}\Delta \mathrm{G} \\
(\mathrm{kcal} / \mathrm{mol})\end{array}$ & $\mathrm{Ki}$ & \multicolumn{2}{|c}{ Ligan-Receptor Interactions } \\
\cline { 5 - 7 } & Azithromycin & $\mathrm{CC}$ & -5.16 & $\begin{array}{c}165.41 \\
\mathrm{uM}\end{array}$ & - & Van der Waals \\
\hline 2 & Favipiravir & $\mathrm{CC}$ & -4.32 & $\begin{array}{c}678.11 \\
\mathrm{uM}\end{array}$ & $\begin{array}{c}\text { Asn142, Gly 143, } \\
\text { Cys145, Ser144 }\end{array}$ & Leu141, Phe140 \\
\hline 3 & Lopinavir & $\mathrm{CC}$ & -9.87 & $58.65 \mathrm{nM}$ & & $\begin{array}{c}\text { Ala191, Thr190, Met 165, } \\
\text { Gln189, His164, Arg188, } \\
\text { Asp187, Met49, His 163, } \\
\text { Leu141, Phe140, Asn 142, } \\
\text { Gly170, Leu167 }\end{array}$ \\
\hline 4 & Nelfinavir & $\mathrm{CC}$ & -9.86 & $59.52 \mathrm{nM}$ & $\begin{array}{c}\text { Glu166, Gln189, } \\
\text { Phe140, His 164 }\end{array}$ & $\begin{array}{c}\text { Thr190, Ala191, Gln192, } \\
\text { Arg188, Gly143, Ser144, } \\
\text { Leu141, His172 }\end{array}$ \\
\hline
\end{tabular}


RASĀYAN J. Chem.

Vol. 14 | No. 3 |1469-1481| July - September | 2021

\begin{tabular}{|c|c|c|c|c|c|c|}
\hline 5 & Oseltamivir & $\mathrm{CC}$ & -7.61 & $2.62 \mathrm{uM}$ & Glu166, Gln189 & $\begin{array}{c}\text { Thr190, Gln192, Arg188, } \\
\text { Asp187, His141, Tyr54, Met49 }\end{array}$ \\
\hline 6 & Remdesivir & $\mathrm{CC}$ & -8.38 & $\begin{array}{c}718.20 \\
\mathrm{nM}\end{array}$ & $\begin{array}{l}\text { Gln189, Ser46, } \\
\text { Leu141, Asn } 142\end{array}$ & $\begin{array}{c}\text { Asp187, His164, Glu166, } \\
\text { Phe140, Ser144, Gly143, } \\
\text { Asn142, Thr45 }\end{array}$ \\
\hline 7 & Ritonavir & $\mathrm{CC}$ & -8.82 & $\begin{array}{c}342.66 \\
\mathrm{nM}\end{array}$ & $\begin{array}{l}\text { Glu166, Gln189, } \\
\text { Met165, Arg188, }\end{array}$ & $\begin{array}{c}\text { Leu167, Gln192, Val186, } \\
\text { Thr190, His164, His41, Tyr54, } \\
\text { Asp187 }\end{array}$ \\
\hline 8 & Amentoflavone & $\mathrm{PC}$ & -10.68 & $14.93 \mathrm{nM}$ & $\begin{array}{l}\text { Glu166, Arg188, } \\
\text { Phe140, His 163, } \\
\text { Cys145 }\end{array}$ & $\begin{array}{c}\text { Pro168, Thr190, Val186, } \\
\text { Gln192, His172, Gln189, } \\
\text { Leu141, Ser144, Asn142, } \\
\text { Gly143, Leu27 } \\
\end{array}$ \\
\hline 9 & Garciniaflavone A & NDC & -8.17 & $1.03 \mathrm{uM}$ & $\begin{array}{l}\text { Glu166, His } 163 \text {, } \\
\text { Gly143, Ser144, } \\
\text { Cys145 }\end{array}$ & $\begin{array}{l}\text { Arg188, His172, Phe140, } \\
\text { Leu141, Leu27, Asn142, } \\
\text { Gln189, Leu167, Pro168, His } \\
164\end{array}$ \\
\hline 10 & Garciniaflavone B & $\mathrm{NDC}$ & -8.8 & $\begin{array}{c}356.56 \\
\mathrm{nM}\end{array}$ & Met165, Gly 143 & $\begin{array}{c}\text { Ser144, Leu141, Phe140, } \\
\text { His172, Asn142, Leu27, } \\
\text { Asp187, Arg188, Val186, } \\
\text { Gln192, His164, Glu166 }\end{array}$ \\
\hline 11 & Garciniaflavone C & NDC & -11.02 & $8.32 \mathrm{nM}$ & \begin{tabular}{|c|} 
Gly143, Leu141, \\
His163, Gln192, \\
Thr190 \\
\end{tabular} & $\begin{array}{c}\text { Asn142, Ser144, Phe140, Met49, } \\
\text { His164, Asp187, Arg188, } \\
\text { Ala191, Leu167 }\end{array}$ \\
\hline 12 & Garciniaflavone D & NDC & -11.38 & $4.57 \mathrm{nM}$ & $\begin{array}{l}\text { His163, Leu141, } \\
\text { Gln192, Thr190 }\end{array}$ & $\begin{array}{c}\text { Ala191, Leu167, Arg188, } \\
\text { His172, Asp187, His164, } \\
\text { Glu166, Phe140, Met49, Ser144, } \\
\text { Asn142, Gly143 }\end{array}$ \\
\hline 13 & Garciniaflavone E & NDC & -9.85 & $59.75 \mathrm{nM}$ & $\begin{array}{c}\text { Gly } 143, \text { Ser } 144 \\
\text { His } 163\end{array}$ & $\begin{array}{l}\text { Leu141, Asn142, His172, } \\
\text { Glu166, Met165, His172, } \\
\text { Phe140, His164, Arg188, } \\
\text { Thr190, Ala191, Gln192, } \\
\text { Leu167 }\end{array}$ \\
\hline 14 & Garciniaflavone $\mathbf{F}$ & NDC & -9.65 & $85.07 \mathrm{nM}$ & $\begin{array}{c}\text { Glu166, Gly143, } \\
\text { Cys145, Ser144, } \\
\text { His } 163\end{array}$ & $\begin{array}{l}\text { Met49, Leu141, Asn142, } \\
\text { His164, Arg188, Thr190, } \\
\text { Ala191, His172, Phe140 }\end{array}$ \\
\hline 15 & Pyranoamentoflavone & NDC & -10.28 & $29.01 \mathrm{nM}$ & $\begin{array}{l}\text { Glu166, Asn142, } \\
\text { Cys145, His163, } \\
\text { Leu141 }\end{array}$ & $\begin{array}{c}\text { Ala191, Thr190, Gln189, } \\
\text { Arg188, Tyr54, Asp187, His164, } \\
\text { Ser144, Phe140, Leu167, } \\
\text { Gln192. }\end{array}$ \\
\hline 16 & $\begin{array}{c}\text { Taiwanhomoflavone } \\
\text { A }\end{array}$ & $\mathrm{NDC}$ & -10.56 & $18.22 \mathrm{nM}$ & $\begin{array}{l}\text { Phe140, His 163, } \\
\text { Leu141, Cys145, } \\
\text { Sser144, Gln189 }\end{array}$ & $\begin{array}{l}\text { His172, Met165, Ala191, } \\
\text { Thr190, Gln192, Arg188, } \\
\text { Asn142, Gly143, Leu27 }\end{array}$ \\
\hline 17 & $\begin{array}{c}\text { Taiwanhomoflavone } \\
\text { B }\end{array}$ & NDC & -8.67 & $\begin{array}{c}442.84 \\
\mathrm{nM}\end{array}$ & $\begin{array}{c}\text { Glu166, His 163, } \\
\text { Ser144, Leu141, } \\
\text { Cys145 } \\
\end{array}$ & $\begin{array}{c}\text { Met165, Phe140, Asn142, } \\
\text { Gly143, Leu27, His41, Gly143, } \\
\text { Met49, Leu167, Pro168 }\end{array}$ \\
\hline 18 & H1 & $\mathrm{ADC}$ & -9.49 & $\begin{array}{c}110.85 \\
\mathrm{nM}\end{array}$ & $\begin{array}{l}\text { His 164, Cys145, } \\
\text { Thr190, Pro168 }\end{array}$ & $\begin{array}{c}\text { Ala191, Gln189, Leu167, } \\
\text { Arg188, Asp187, Tyr54, Met49, } \\
\text { Asn142, Gly143, His163, } \\
\text { Glu166 }\end{array}$ \\
\hline 19 & $\mathrm{H} 2$ & $\mathrm{ADC}$ & -11.89 & $1.92 \mathrm{nM}$ & Thr190 & $\begin{array}{c}\text { Glu166, Leu167, Gln192, } \\
\text { Pro168, Ala191, Arg188, } \\
\text { His164, Asp187, Tyr54, Asn142, } \\
\text { Gln189, Ser46 }\end{array}$ \\
\hline
\end{tabular}


RASĀYAN J. Chem.

Vol. 14 | No. 3 |1469-1481| July - September | 2021

\begin{tabular}{|c|c|c|c|c|c|c|}
\hline 20 & H3 & $\mathrm{ADC}$ & -10.99 & $8.80 \mathrm{nM}$ & $\begin{array}{l}\text { Glu166, Gln } 189, \\
\text { Cys145 }\end{array}$ & $\begin{array}{c}\text { Leu50, Ala191, Thr190, Gln192, } \\
\text { His163, Asn142, Arg188, } \\
\text { His164, Asp187, Tyr54 }\end{array}$ \\
\hline 21 & H4 & $\mathrm{ADC}$ & -9.92 & $53.91 \mathrm{nM}$ & $\begin{array}{c}\text { Cys145, His 164, } \\
\text { Thr190 }\end{array}$ & $\begin{array}{c}\text { His163, Glu166, Gln189, } \\
\text { Pro168, Ala191, Leu167, } \\
\text { Arg188, Aspp187, Tyr54, Met49 }\end{array}$ \\
\hline 22 & H5 & $\mathrm{ADC}$ & -9.32 & $\begin{array}{c}146.54 \\
\mathrm{nM}\end{array}$ & $\begin{array}{c}\text { Asn142, Leu141, } \\
\text { Cys145, His163, } \\
\text { Pro168 } \\
\end{array}$ & $\begin{array}{c}\text { Thr190, Ala191, Gln192, } \\
\text { Leu167, Arg188, Tyr54, Glu166, } \\
\text { His164, Ser144, His172, Phe140 }\end{array}$ \\
\hline 23 & H6 & $\mathrm{ADC}$ & -12.7 & $\begin{array}{c}490.73 \\
\text { pM }\end{array}$ & Glu166 & $\begin{array}{c}\text { Thr190, Gln189, His164, } \\
\text { Asp187, Tyr54, Arg188, } \\
\text { Met165, His163, Asn142, } \\
\text { Phe140, Gly143, Leu141, Ser144 }\end{array}$ \\
\hline 24 & S1 & $\mathrm{ADC}$ & -10.85 & $11.14 \mathrm{nM}$ & $\begin{array}{c}\text { Glu166, Phe140, } \\
\text { Cys45, Leu141, } \\
\text { Ser44, Gly43, } \\
\text { Thr190 } \\
\end{array}$ & $\begin{array}{c}\text { His172, His163, Asn142, Ser46, } \\
\text { Gln189, Arg188, Gln192, } \\
\text { Leu167 }\end{array}$ \\
\hline 25 & $\mathbf{S 2}$ & $\mathrm{ADC}$ & -10.59 & $17.33 \mathrm{nM}$ & $\begin{array}{c}\text { Glu166, Gln189, } \\
\text { Phe140, Leu141, } \\
\text { Ser144, Cys45, } \\
\text { Gly143 } \\
\end{array}$ & $\begin{array}{c}\text { Thr190, Ala191, Gln192, } \\
\text { Arg188, Met49, Asn142, His163 }\end{array}$ \\
\hline 26 & S3 & $\mathrm{ADC}$ & -10.79 & $12.30 \mathrm{nM}$ & $\begin{array}{l}\text { Glu166, Gln189, } \\
\text { Gln192, Thr190 }\end{array}$ & $\begin{array}{c}\text { Asn142, Ser144, Gly1143, } \\
\text { Leu27, His164, Arg188, Ala191 } \\
\text { Leu167 }\end{array}$ \\
\hline 27 & S4 & $\mathrm{ADC}$ & -10.82 & $11.68 \mathrm{nM}$ & $\begin{array}{l}\text { Gln189, Gln192, } \\
\text { Arg188, Leu141 }\end{array}$ & $\begin{array}{c}\text { Asn142, Phe40, Ser144, His163, } \\
\text { His164, Asp187, Tyr54, Val186, } \\
\text { Thr190, Ala911, Leu167 }\end{array}$ \\
\hline 28 & S5 & $\mathrm{ADC}$ & -10.17 & $35.36 \mathrm{nM}$ & $\begin{array}{l}\text { Gln92, Arg188, } \\
\text { Cys145, Leu141, } \\
\text { His163, Asn } 1142\end{array}$ & $\begin{array}{c}\text { Phe140, Ser144, His164, Met49, } \\
\text { Gln189, Val186, Thr190, } \\
\text { Leu1167, Ala191 } \\
\end{array}$ \\
\hline 29 & S6 & $\mathrm{ADC}$ & -10.7 & $14.34 \mathrm{nM}$ & $\begin{array}{l}\text { Asn142, Gln192, } \\
\text { Arg88, Cys145, } \\
\text { Leu1141 }\end{array}$ & $\begin{array}{c}\text { His172, Phe140, Ser144, His163, } \\
\text { His164, Tyr54, Asp187, Met49, } \\
\text { Val186, Gln189, Thr190, } \\
\text { Ala191, Leu167 } \\
\end{array}$ \\
\hline 30 & S7 & $\mathrm{ADC}$ & -10.44 & $22.12 \mathrm{nM}$ & $\begin{array}{l}\text { Glu166, Gln189, } \\
\text { Gln1192, Thr190 }\end{array}$ & $\begin{array}{c}\text { Leu167, Ala191, His164, Met49, } \\
\text { Asn142, Leu27, Gly143 } \\
\end{array}$ \\
\hline 31 & S8 & $\mathrm{ADC}$ & -9.33 & $\begin{array}{c}144.92 \\
\mathrm{nM}\end{array}$ & $\begin{array}{l}\text { Glu166, Met165, } \\
\text { His 164, His41 }\end{array}$ & $\begin{array}{c}\text { Arg188, Asp187, Gln189, } \\
\text { Pro168, His172, Phe140, } \\
\text { Leu141, Ser144, Asn142, } \\
\text { Gly143, Leu27 }\end{array}$ \\
\hline 32 & S9 & $\mathrm{ADC}$ & -9.29 & $\begin{array}{c}154.45 \\
\mathrm{nM}\end{array}$ & $\begin{array}{l}\text { Glu166, Thr190, } \\
\text { Gln190 }\end{array}$ & $\begin{array}{c}\text { Leu167, Asn142, Gly143, } \\
\text { Cys145, Met49, His164, Arg188, } \\
\text { Ala191 }\end{array}$ \\
\hline 33 & $\mathrm{~S} 10$ & $\mathrm{ADC}$ & -11.08 & $7.55 \mathrm{nM}$ & $\begin{array}{l}\text { Glu166, Thr190, } \\
\text { Gln1192 }\end{array}$ & $\begin{array}{c}\text { Ser46, Leu50, Thr25, Gly143, } \\
\text { Asn142, His164, Ala1911, } \\
\text { Leu167 }\end{array}$ \\
\hline 34 & $\mathrm{~S} 11$ & $\mathrm{ADC}$ & -10.5 & $20.19 \mathrm{nM}$ & $\begin{array}{c}\text { Glu166, Gln189, } \\
\text { Thr190, Gln192, } \\
\text { His41 } \\
\end{array}$ & $\begin{array}{l}\text { Leu67, Ala191, Arg188, His164, } \\
\text { Leu27, Gly143, Ser144, Asn142 }\end{array}$ \\
\hline 35 & $\mathrm{~S} 12$ & $\mathrm{ADC}$ & -10.88 & $10.61 \mathrm{nM}$ & $\begin{array}{l}\text { Glu166, Thr190, } \\
\text { Gln192 }\end{array}$ & $\begin{array}{c}\text { Leu67, Ala191, Arg188, } \\
\text { Asp187, His41, Leu27, Ser144, } \\
\text { Asn42, Gly43 }\end{array}$ \\
\hline
\end{tabular}


RASĀYAN J. Chem.

Vol. 14 | No. 3 |1469-1481| July - September | 2021

\begin{tabular}{|c|c|c|c|c|c|c|}
\hline 36 & S13 & $\mathrm{ADC}$ & -10.23 & $31.79 \mathrm{nM}$ & $\begin{array}{l}\text { Glu166, Gln89, } \\
\text { Thr190, Gln192 }\end{array}$ & $\begin{array}{c}\text { Asn142, Met49, Gly143, His164, } \\
\text { Arg188, Ala191, Leu167 } \\
\end{array}$ \\
\hline 37 & S14 & $\mathrm{ADC}$ & -10.48 & $20.94 \mathrm{nM}$ & $\begin{array}{c}\text { Phe140, Asn } 142, \\
\text { Gln192, Arg 188, } \\
\text { Cys145, Leu141, } \\
\text { His } 163 \\
\end{array}$ & $\begin{array}{c}\text { Leu67, Ala191, Thr190, Val86, } \\
\text { Gln189, Met49, Asp187, His164, } \\
\text { Ser144 }\end{array}$ \\
\hline 38 & S15 & $\mathrm{ADC}$ & -10.71 & $14.17 \mathrm{nM}$ & $\begin{array}{l}\text { Phe140, Asn142, } \\
\text { Gln192, Arg188 }\end{array}$ & $\begin{array}{c}\text { Ser144, His163, His164, Tyr54, } \\
\text { Mer49, Gln189, Val186, Thr190, } \\
\text { Ala191, Leu167 }\end{array}$ \\
\hline 39 & S16 & $\mathrm{ADC}$ & -10.57 & $17.98 \mathrm{nM}$ & $\begin{array}{l}\text { Glu66, Gln189, } \\
\text { Thr190, Gln192 }\end{array}$ & $\begin{array}{l}\text { Asn142, Gly143, His164, } \\
\text { Ala191, Leu167 }\end{array}$ \\
\hline 40 & S17 & $\mathrm{ADC}$ & -9.78 & $67.74 \mathrm{nM}$ & $\begin{array}{l}\text { Glu166, Met165, } \\
\text { His164, His41 }\end{array}$ & $\begin{array}{c}\text { Arg188, Gln189, Asp187, } \\
\text { Pro168, His172, Phe140, } \\
\text { Leu141, Ser144, Asn142, } \\
\text { Gly143, Leu27 } \\
\end{array}$ \\
\hline 41 & S18 & $\mathrm{ADC}$ & -9.38 & $\begin{array}{c}132.08 \\
\mathrm{nM}\end{array}$ & $\begin{array}{l}\text { Glu166, Thr190, } \\
\text { Gln192, Gly143 }\end{array}$ & $\begin{array}{c}\text { Gln189, Leu167, Ala191, } \\
\text { Arg188, His164, Met49, Cys145, } \\
\text { Asn142 }\end{array}$ \\
\hline 42 & S19 & $\mathrm{ADC}$ & -11.14 & $6.80 \mathrm{nM}$ & $\begin{array}{l}\text { Glu166, Thr190, } \\
\text { Gln192 }\end{array}$ & $\begin{array}{l}\text { Asn142, Gly143, Thr25, Leu27, } \\
\text { His164, Leu167, Ala191, Leu50, } \\
\text { Ser46, Gln89 }\end{array}$ \\
\hline 43 & S20 & $\mathrm{ADC}$ & -10.41 & $23.35 \mathrm{nM}$ & $\begin{array}{l}\text { Glu166, Gln189, } \\
\text { Ser144, Phe140 }\end{array}$ & $\begin{array}{l}\text { Thr190, Ala191, Gln192, } \\
\text { Arg188, Asn1142, Gly143, } \\
\text { Leu141, His163, His172 }\end{array}$ \\
\hline 44 & S21 & $\mathrm{ADC}$ & -10.62 & $16.44 \mathrm{nM}$ & $\begin{array}{l}\text { Glu166, Gln189, } \\
\text { Phe140, Ser144 }\end{array}$ & $\begin{array}{l}\text { Thr190, Ala191, Gln192, } \\
\text { Arg188, Asn1142, Gly143, } \\
\text { Leu141, His163, His172 }\end{array}$ \\
\hline 45 & S22 & $\mathrm{ADC}$ & -10.46 & $21.55 \mathrm{nM}$ & $\begin{array}{l}\text { Glu166, Asn142, } \\
\text { Phe140, His 172, } \\
\text { Cys145 }\end{array}$ & $\begin{array}{c}\text { Ser144, His163, Leu141, } \\
\text { Gln189, Asp187, Tyr54, Arg188, } \\
\text { Thr25, His164, Met49, Gly143 }\end{array}$ \\
\hline 46 & S23 & $\mathrm{ADC}$ & -9.99 & $47.94 \mathrm{nM}$ & $\begin{array}{c}\text { Phe140, Leu141, } \\
\text { His163, Cys145, } \\
\text { Gln192, Arg188, } \\
\text { Thr190 } \\
\end{array}$ & $\begin{array}{l}\text { Ser144, Asn142, Leu167, } \\
\text { Ala191, Val186, Gln189, } \\
\text { Asp187, Met49, His164 }\end{array}$ \\
\hline 47 & S24 & $\mathrm{ADC}$ & -9.62 & $89.00 \mathrm{nM}$ & $\begin{array}{l}\text { Asn142, Gly143, } \\
\text { Cys145, Arg188, } \\
\text { Gln192, Thr'190, } \\
\text { Met49 }\end{array}$ & $\begin{array}{c}\text { Glu166, Gln189, Leu167, } \\
\text { Pro168, Ala191, Val186, } \\
\text { Asp187, Pro52, Tyr54, His1164, } \\
\text { His163, Ser144, Phe140 }\end{array}$ \\
\hline 48 & S25 & $\mathrm{ADC}$ & -10.37 & $24.96 \mathrm{nM}$ & $\begin{array}{l}\text { Glu166, Gln189, } \\
\text { Thr190, Gln192, }\end{array}$ & $\begin{array}{l}\text { Leu27, Gly43, Met49, Asn142, } \\
\text { Leu67, Ala191, His164 }\end{array}$ \\
\hline 49 & S26 & $\mathrm{ADC}$ & -9.27 & $\begin{array}{c}159.57 \\
\mathrm{nM}\end{array}$ & $\begin{array}{l}\text { Thr190, Gln192, } \\
\text { Cys145, His164, } \\
\text { Leu141 }\end{array}$ & $\begin{array}{c}\text { Leu167, Ser144, Gly143, } \\
\text { Asn142, Gln189, Arg188, } \\
\text { Ala191 }\end{array}$ \\
\hline 50 & S27 & $\mathrm{ADC}$ & -9.37 & $\begin{array}{c}136.54 \\
\mathrm{nM}\end{array}$ & $\begin{array}{c}\text { Glu166, Gln189, } \\
\text { Thr190, Gly143, } \\
\text { Cys145, Ser144, } \\
\text { Phe140 } \\
\end{array}$ & $\begin{array}{l}\text { Leu167, Pro168, Gln192, } \\
\text { Arg188, Asn142, His 163, } \\
\text { Leu141, His172 }\end{array}$ \\
\hline 51 & S28 & $\mathrm{ADC}$ & -10.88 & $10.67 \mathrm{nM}$ & $\begin{array}{l}\text { Glu166, Gln189, } \\
\text { Ser144, Phe140 }\end{array}$ & $\begin{array}{c}\text { Ala1191, Thr190, Gln192, } \\
\text { Ser46, Asn142, Gly143, His163, } \\
\text { Leu141, His172 }\end{array}$ \\
\hline
\end{tabular}


RASĀYAN J. Chem.

Vol. 14 | No. 3 |1469-1481| July - September | 2021

\begin{tabular}{|c|c|c|c|c|c|c|}
\hline 52 & S29 & $\mathrm{ADC}$ & -13.04 & $\begin{array}{c}274.93 \\
\mathrm{pM}\end{array}$ & $\begin{array}{l}\text { Glu166, Asn142, } \\
\text { Met49, Phe140, } \\
\text { Cys145, Ser144 }\end{array}$ & $\begin{array}{l}\text { Gly143, Ser46, Pro168, Thr190, } \\
\text { Gln192, Arg188, Tyr54, Asp186, } \\
\text { His172, His163, His164, Leu141 }\end{array}$ \\
\hline 53 & S30 & $\mathrm{ADC}$ & -12.83 & $\begin{array}{l}390.94 \\
\mathrm{pM}\end{array}$ & $\begin{array}{l}\text { Glu166, Arg188, } \\
\text { Asn142, His163, } \\
\text { Cys145 }\end{array}$ & $\begin{array}{c}\text { Pro168, Thr190, Gln192, } \\
\text { Asp187, His164, Ser144, } \\
\text { Phe140, Leu141, Leu27, Gly143, } \\
\text { Gln189 }\end{array}$ \\
\hline 54 & S 31 & $\mathrm{ADC}$ & -12.33 & $\begin{array}{l}909.24 \\
\mathrm{pM}\end{array}$ & $\begin{array}{l}\text { Glu166, Phe140, } \\
\text { Asn142, Gly143 }\end{array}$ & $\begin{array}{c}\text { Thr25, Ser144, His163, His164, } \\
\text { His172, Asp187, Tyr54, Arg188, } \\
\text { Gln189, Leu141 }\end{array}$ \\
\hline 55 & S32 & $\mathrm{ADC}$ & -10.4 & $23.79 \mathrm{nM}$ & $\begin{array}{l}\text { Glu166, Gln192, } \\
\text { Arg188, His163, } \\
\text { Leu141, Cys145 }\end{array}$ & $\begin{array}{c}\text { His164, Gln189, Val186, } \\
\text { Thr190, Ala191, Leu167, } \\
\text { His172, Asn142, Phe140, Ser144 }\end{array}$ \\
\hline 56 & S33 & $\mathrm{ADC}$ & -11.1 & $7.26 \mathrm{nM}$ & $\begin{array}{l}\text { Gln1189, Thr190, } \\
\text { Gln1192, } \\
\text { Arg188, Asn142, } \\
\text { Gly143, His41, } \\
\text { Ser144 }\end{array}$ & $\begin{array}{l}\text { Leu27, Tyr54, Pro52, Asp187, } \\
\text { Val186, Leu167, Ala191, } \\
\text { Pro168, Glu166, Leu141 }\end{array}$ \\
\hline 57 & S34 & $\mathrm{ADC}$ & -11.37 & $4.62 \mathrm{nM}$ & $\begin{array}{c}\text { Glu166, Gln89, } \\
\text { Cys145, His41, } \\
\text { Leu141, Gly143, } \\
\text { Ser144 } \\
\end{array}$ & $\begin{array}{c}\text { Ala191, Thr190, Arg188, } \\
\text { Gln1192, His164, Met49, Thr25, } \\
\text { Leu27, Asn42, His163, Phe140, } \\
\text { Leu167, His172 } \\
\end{array}$ \\
\hline 58 & S35 & $\mathrm{ADC}$ & -10.82 & $11.68 \mathrm{nM}$ & $\begin{array}{c}\text { Thr190, Gln92, } \\
\text { Pro168, Gly143, } \\
\text { Cys145, Ser144, } \\
\text { His } 163 \\
\end{array}$ & $\begin{array}{c}\text { Asp187, Arg188, Ala191, } \\
\text { Gln189, Leu167, Asn142, } \\
\text { Glu166, His41, His172, Phe140, } \\
\text { Leu141 } \\
\end{array}$ \\
\hline 59 & S36 & $\mathrm{ADC}$ & -10.99 & $8.78 \mathrm{nM}$ & $\begin{array}{l}\text { Glu166, Phe140, } \\
\text { His163, Ser144, } \\
\text { Asn142 }\end{array}$ & $\begin{array}{c}\text { Asp187, Gln192, Arg 188, } \\
\text { Thr190, Pro168, Gln189, Ser46, } \\
\text { Gly143, Leu141, His172 }\end{array}$ \\
\hline
\end{tabular}

Where: CC- Comparison Compound, NDC- Natural Derivative Compound, ADC- Artificial Derivative Compound, $\Delta \mathrm{G}-$ Gibbs Energy, Nm- nanomolar, pM- picomolar.

However, the key amino acid residue in the pocket of 6LU7 and the 3D structure of the active center of proteases are also important parameters to be further analyzed. ${ }^{6}$ Table-2 shows that S29 has a strong binding at catalytic amino acid Cys 145 and also four other strong bindings to the surrounding amino acid sequences at $\mathrm{M}^{\text {pro2 }}$ active site, suggesting that $\mathrm{S} 29$ potentially inhibits $\mathrm{M}^{\text {pro2 }}$ enzymatic activity better than $\mathrm{N}$.

Table-2: Comparison of Hydrogen Bond Distances in the Interaction between $\mathrm{M}^{\text {pro2 }}$ Inhibitors and their Binding Target in $\mathrm{M}^{\text {pro2 }}$ Active Site Amino Acid Sequences

\begin{tabular}{|c|c|c|c|c|c|c|c|c|}
\hline \multirow{2}{*}{$\begin{array}{c}\mathrm{M}^{\mathrm{pro2}} \\
\text { active } \\
\text { sites }\end{array}$} & \multicolumn{2}{|c|}{$\begin{array}{c}\mathrm{N} 3 \\
\text { (native ligand) }\end{array}$} & \multicolumn{2}{|c|}{$\begin{array}{l}\text { Remdesivir } \\
\text { (comparison } \\
\text { compound) }\end{array}$} & \multicolumn{2}{|c|}{$\begin{array}{l}\text { Amentoflavone } \\
\text { (lead compound) }\end{array}$} & \multicolumn{2}{|c|}{$\begin{array}{c}\text { S29 } \\
\text { (derivative } \\
\text { compound) }\end{array}$} \\
\hline & $\mathrm{R}(\AA)^{*}$ & Strength & $\mathrm{R}(\AA)^{*}$ & Strength & $\begin{array}{r}\mathrm{R} \\
(\AA)^{*}\end{array}$ & Strength & $\mathrm{R}(\AA)^{*}$ & Strength \\
\hline Phe140 & - & - & - & - & - & - & 2.41 & Strong \\
\hline Asn142 & - & - & - & - & - & - & 2.11 & Strong \\
\hline Gly 143 & 2.9 & Moderate & - & - & 2.01 & & - & - \\
\hline Cys145 & 1.8 & Strong & 3.89 & Weak & 4.10 & $\begin{array}{l}\text { Electrostati } \\
\mathrm{c}\end{array}$ & 2.06 & Strong \\
\hline His 163 & 2.4 & Strong & - & - & 2.13 & Strong & - & - \\
\hline His 164 & 2.8 & Moderate & - & - & 3.07 & Moderate & - & - \\
\hline Glu166 & 2.8 & Moderate & 2.37 & Strong & - & - & 2.21 & Strong \\
\hline
\end{tabular}


RASĀYAN J. Chem.

Vol. 14 | No. 3 |1469-1481| July - September | 2021

\begin{tabular}{l|l|l|r|l|r|l|l|l}
\hline Gln189 & 2.9 & Moderate & 1.95 & Strong & - & - & - \\
\hline Thr190 & 2.8 & Moderate & - & - & 1.95 & Strong & - & - \\
\hline
\end{tabular}

Highlighted bold: Catalytic amino acid of the active site of M Mro2 $^{\text {PDB ID: 6LU7) }}$

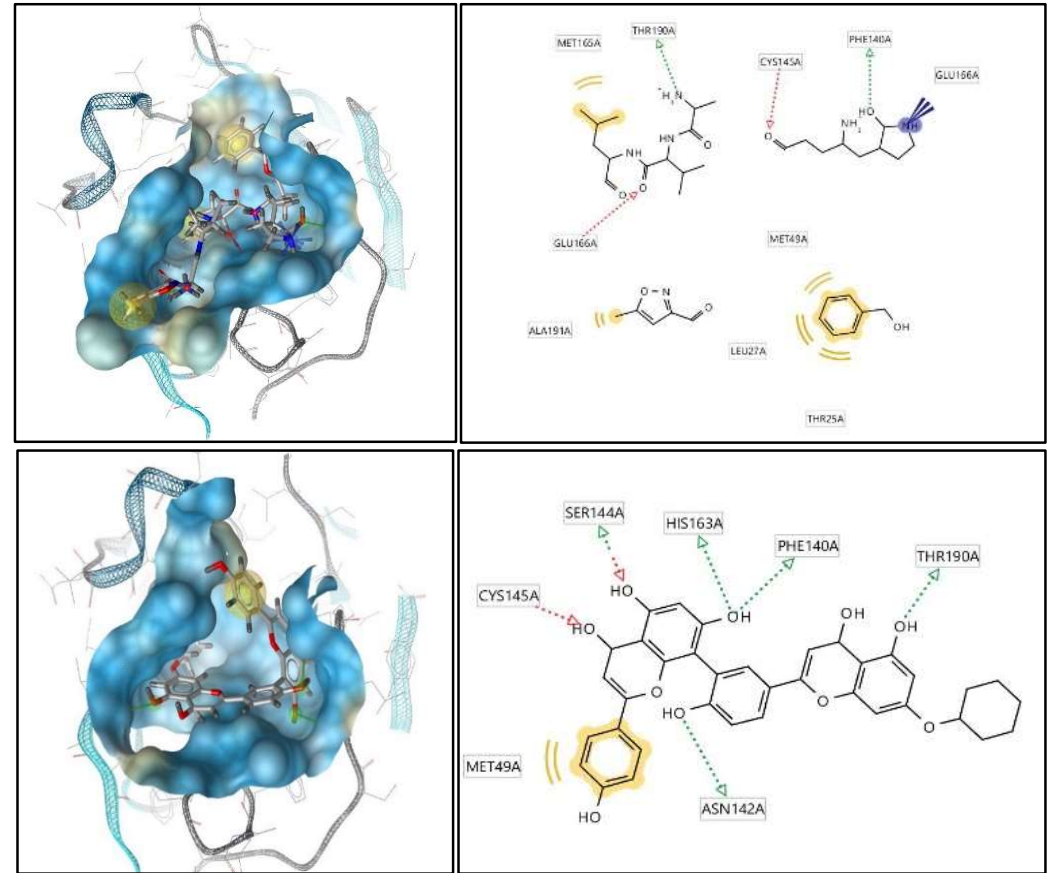

Fig.-3: Molecular Interaction between $\mathrm{M}^{\text {pro2 }}$ and N3 (as a Native Ligand) (A) and S29 (B), the Best-docked Compound

\section{D Ligan-Based Pharmacophore Modeling}

Pharmacophores modeling was carried out to evaluate the similarity of the bonds of a test ligand (amentoflavone and its derivatives) with previously known active ligand at $\mathrm{M}^{\text {pro2 }}$ active site, in particular at the catalytic amino acid (Cys145). ${ }^{22} \mathrm{~A}$ 3D ligan-based pharmacophore modeling determines the ligand's part responsible for biological activity. A validated 3D ligan-based pharmacophore model (by an algorithm in LigandScout 4.1 Advanced) was utilized to virtually screen amentoflavone and its derivatives. Validation was performed using an active set (100 ligands that are active at certain receptors with known $\mathrm{IC}_{50}$ value) and a decoy set (400 ligands with a similar structure to the active set but inactive against the receptor) obtained from http://dude.docking.org. ${ }^{22}$

The best 33623 hits were obtained from screening; all were from the active set (Fig.-4). The enrichment factor value of $100 \%\left(\mathrm{EF}_{100 \%}\right)$ of 1.1 and area under curve $100 \%\left(\mathrm{AUC}_{100 \%}\right)$ of 0.60 were achieved. The $\mathrm{X}-$ axis on the receiver operating characteristic (ROC) curve is the speed of the active composition and the $\mathrm{Y}$ velocity is the speed of the feed composition. The ROC curve follows a diagonal line representing a randomized classification model unable to distinguish between active and decoy ligands. The pharmacophores modeling has been properly validated because it has a value $\left(\mathrm{AUC}_{100 \%}\right)$ of $>0.5 .{ }^{22}$

3D pharmacophore modeling of Amentoflavone and its derivatives are reported in Table-S5 (showed as pharmacophore-fit score) that can measure the geometric fit of the mode of interactions of a ligand to expressed model. A higher fit score, a better similarity to the expressed model. Therefore, the ligands that fit the pharmacophore model show activity at 6LU7 because not all of the mode of interaction of the model will be matched any two features that omitted during the virtual screening process. In this case, mode of interactions that do not be matched showed in lower pharmacophore fit scores. The pharmacophore-fit scores of amentoflavone and its derivatives (35.69 to 45.01) were all higher than the parent compound (35.71 to 37.65), and its lead compound, amentoflavone (35.69). S12 has the best pharmacophore fit scores, indicates that its chemical interaction aligned best to the mode of interaction of the database of actives model. 
RASĀYAN J. Chem.

Vol. 14 | No. 3 |1469-1481| July - September | 2021

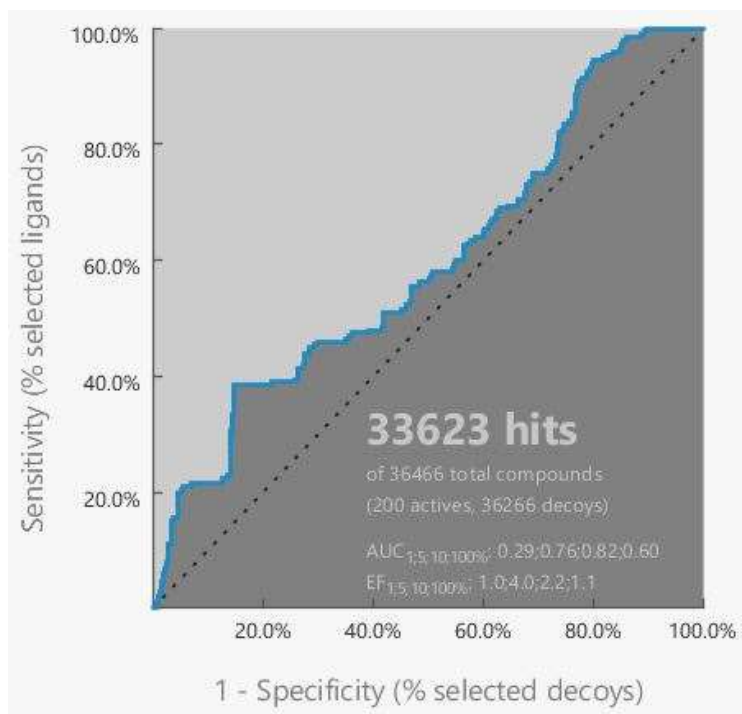

Fig.-4: ROC Curve on the Validation of the Pharmacophores Modeling Method

ADME Prediction

The parameters used in the ADME prediction are human intestinal absorption (HIA), Caco-2, plasma protein binding (PPB), and blood-brain barrier (BBB). HIA shows the amount of a compound that can be absorbed by the human intestine. Caco-2 cells (human colon adenocarcinoma) are substitutes for human intestinal epithelial cells used to estimate in vitro drug permeability. PPB was evaluated by simulating the attachment to plasma protein (mainly albumin). Anti-SARS-CoV-2 drugs are not designed to penetrate the BBB to avoid psychotropic side effects, ${ }^{23}$ hence drugs that penetrate BBB will be excluded.

Table-S4 (Supplementary Data) shows that all amentoflavone derivative compounds have the potential to be absorbed in the intestine and have better permeability potential compared to remdesivir. Various amentoflavone derivative compounds have a higher PPB value than comparative drugs. As a note, PPB value $>99 \%$ may still be tolerated since free drug concentrations can still be provided. ${ }^{24}$ However, compounds H2, H3, H6, S1, S10, S19, S28, and S34 have to be excluded from potential $\mathrm{M}^{\text {pro2 }}$ inhibitors since they did not satisfy BBB parameters.

Recently, amentoflavone has been reported as among top-ranking compounds whose stronger binding against $\mathrm{M}^{\mathrm{pro} 2}$ in comparison to millions of natural compounds, FDA-approved drugs, and drug candidates in clinical trials in several molecular docking studies (smallest $\Delta \mathrm{G}$ : $-9.28 \mathrm{kcal} / \mathrm{mol}$ ). ${ }^{25-27}$ In the current study, several amentoflavone derivative compounds have been subject to molecular docking simulations against $\mathrm{M}^{\mathrm{pro2}}$. Not only these efforts might reduce mutagenic and toxicity potentials of the native amentoflavone, but an approximately 50 times stronger binding affinity was also potentially achieved ( $\mathrm{Ki}$ of S29 and amentoflavone, $275 \mathrm{pM}$ and $15 \mathrm{nM}$, respectively).

The main characteristics of potent $\mathrm{M}^{\mathrm{pro2}}$ inhibitors are the presence of multiple bi- and monocyclic rings (mostly heterocycles and aromatic), which can make a ligand-target interaction of the $\mathrm{M}^{\mathrm{pro2}}$ active site especially at the catalytic dyad residues (His41 and Cys145). ${ }^{25} \mathrm{~A}$ strong hydrogen bond to Glu166 is also suggested essential for affinity strength during Mpro2 inhibition, as characterized by several potential $\mathrm{M}^{\text {pro2 }}$ inhibitors (including amentoflavone). ${ }^{28}$ Molecularly, S29 as the best-docked compound can fulfill the ideal characteristics as a good $\mathrm{M}^{\mathrm{pro2}}$ inhibitor. This is because $\mathrm{S} 29$ can interact with the catalytic dyad from Mpro2 through the hydroxyl and carbonyl groups on the C4" and C5" atoms and can interact with Glu166 through strong hydrogen bonds in the cyclo-oxy structure on the $\mathrm{C} 1$ which can stabilize the chemical interactions between the ligands and the $\mathrm{M}^{\text {pro2 }}$. Interestingly, S29 does not only feature a strong hydrogen bond to Glu166 but also stabilized by strong hydrogen bonds against several other amino acid residues at the $\mathrm{M}^{\text {pro2 }}$ active site (Table-1, Table-2). A recent study also indicates that amentoflavone is also among the topranking compounds targeting SARS-CoV-2 spike glycoprotein. ${ }^{29}$ If this condition is also fulfilled by S29, its pharmacological benefits will be two-fold; inhibition of viral replication and viral entry, a rare property among antiviral drugs. However, this speculation warrants further investigations. 
RASĀYAN J. Chem.

Vol. 14 | No. 3 |1469-1481| July - September | 2021

\section{CONCLUSION}

In-silico studies revealed 18 (4 natural, 14 artificial) amentoflavone derivative compounds met the criteria as candidates for $\mathrm{M}^{\text {pro2 }}$ specific inhibitor and one compound among them might be formulated as an oral preparation. These compounds may be potentially developed as anti-SARS-CoV-2 drugs.

Associated Content: The supplementary data is available by request to the authors.

\section{ACKNOWLEDGEMENT}

This research was funded by "Hibah Riset Data Pustaka dan Daring (Grants for Literature and Online Research)" (No. 1735/UN6.3.1/LT/2020) from the Directorate of Research, Community Service, and Innovation, Universitas Padjadjaran. The authors are also thankful to the Department of Pharmaceutical Analysis and Medicinal Chemistry, Faculty of Pharmacy, Universitas Padjadjaran.

\section{REFERENCES}

1. WHO, Coronavirus disease (COVID-19) Weekly Epidemiological Update and Weekly Operational Update, World Health Organization (2020), Press Release: 21 April 2021, Available at: https://www.who.int/emergencies/diseases/novel-coronavirus-2019/situation-reports

2. WHO, Draft landscape of COVID-19 Candidate Vaccines, World Health Organization (2020), Press Release: 3 September 2020, Available at: https://www.who.int/publications/m/item/draft-landscape-ofcovid-19-candidate-vaccines

3. Stephanie Nebehay, Emma Farge, and (Reuters), Widespread COVID-19 Vaccinations not expected until mid-2021, WHO says, World News, Geneva, September 4, 2020(2020), Available at: https://www.reuters.com/article/us-health-coronavirus-who-briefing/widespread-covid-vaccinationsnot-expected-until-mid-2021-who-says-idUSKBN25V1B0

4. Y. Jin, H. Yang, W. Ji, W. Wu, S. Chen, W. Zhang, and G. Duan, Viruses 12, 372(2020), https://doi.org/10.3390/v12040372

5. Z. Jin, X. Du, Y. Xu, Y. Deng, M. Liu, Y. Zhao, B. Zhang, X. Li, L. Zhang, and C. Peng, Nature, 582, 1(2020), https://doi.org/10.1038/s41586-020-2223-y

6. P. Eleftheriou, D. Amanatidou, A. Petrou, and A. Geronikaki, Molecules 25, 2529(2020), https://doi.org/10.3390/molecules25112529

7. M. S. Smyth and J. H. Martin, Molecular Pathology 53, 8(2000), https://doi.org/10.1136/mp.53.1.8

8. G. U. Jeong, H. Song, G. Y. Yoon, D. Kim, and Y.-C. Kwon, Frontiers in Microbiology, 11, 1723 (2020), https://doi.org/10.3389/fmicb.2020.01723

9. S. J. Tzotzos, B. Fischer, H. Fischer, and M. Zeitlinger, Critical Care, 24, 516(2020), https://doi.org/10.1186/s13054-020-03240-7

10. WHO, "Solidarity" clinical trial for COVID-19 treatments, World Health Organization (2020), Press Release: July 6, 2020. Available at: https://www.who.int/emergencies/diseases/novel-coronavirus2019/global-research-on-novel-coronavirus-2019-ncov/solidarity-clinical-trial-for-covid-19$\underline{\text { treatments }}$

11. G. Ragia and V. G. Manolopoulos, European Journal of Clinical Pharmacology, 76,1623(2020), https://doi.org/10.1007/s00228-020-02963-4

12. C. Monari, V. Gentile, C. Camaioni, G. Marino, N. Coppola, and V. COVID, Life 10, 146(2020), https://doi.org/10.3390/life10080146

13. S. Yu, H. Yan, L. Zhang, M. Shan, P. Chen, A. Ding, and S. F. Y. Li, Molecules: A Journal of Synthetic Chemistry and Natural Product Chemistry, 22, 299(2017), https://doi.org/10.3390/molecules22020299

14. Y. B. Ryu, H. J. Jeong, J. H. Kim, Y. M. Kim, J. Y. Park, D. Kim, T. T. Nguyen, S. J. Park, J. S. Chang, K. H. Park, M. C. Rho, and W. S. Lee, Bioorganic \& Medicinal Chemistry, 18, 7940 (2010), https://doi.org/10.1016/j.bmc.2010.09.035

15. C. R. P. Cardoso, I. M. d. S. Cólus, C. C. Bernardi, M. Sannomiya, W. Vilegas, and E. A. Varanda, Toxicology 225, 55 (2006), https://doi.org/10.1016/j.tox.2006.05.003

16. C. A. Lipinski, Drug Discovery Today: Technologies 1, 337(2004), https://doi.org/10.1016/j.ddtec.2004.11.007 
RASĀYAN J. Chem.

Vol. 14 | No. 3 |1469-1481| July - September | 2021

17. M. Bzowka, K. Mitusinska, A. Raczynska, A. Samol, J. A. Tuszynski, and A. Gora, International Journal of Molecular Sciences, 21, 3099 (2020), https://doi.org/10.3390/ijms21093099

18. M. Vieth, M. G. Siegel, R. E. Higgs, I. A. Watson, D. H. Robertson, K. A. Savin, G. L. Durst, and P. A. Hipskind, Journal of Medicinal Chemistry, 47, 224 (2004), https://doi.org/10.1021/jm030267j

19. A. Puratchikody, D. Sriram, A. Umamaheswari, and N. Irfan, Chemistry Central Journal, 10, 24 (2016), https://doi.org/10.1186/s13065-016-0169-9

20. A. Tronde, B. Norden, H. Marchner, A. K. Wendel, H. Lennernas, and U. H. Bengtsson, Journal of Pharmaceutical Sciences, 92, 1216 (2003), https://doi.org/10.1002/jps.10386

21. G. A. Jeffrey, "An introduction to hydrogen bonding." Oxford University Press, New York, (1997), https://doi.org/10.1021/ja9756331

22. M. Wieder, U. Perricone, T. Seidel, S. Boresch, and T. Langer, Monatshefte für Chemie/Chemical Monthly, 147, 553 (2016), https://doi.org/10.1007/s00706-016-1674-1

23. T. S. Carpenter, D. A. Kirshner, E. Y. Lau, S. E. Wong, J. P. Nilmeier, and F. C. Lightstone, Biophysical Journal, 107, 630 (2014), https://doi.org/10.1016/j.bpj.2014.06.024

24. W. E. Lindup and M. C. Orme, British Medical Journal (Clinical Research Edition), 282, 212(1981), https://doi.org/10.1136/bmj.282.6259.212

25. O. O. Olubiyi, M. Olagunju, M. Keutmann, J. Loschwitz, and B. Strodel, Molecules, 25 (2020), https://doi.org/10.3390/molecules25143193

26. L. Peterson, COVID-19 and Flavonoids: In-silico Molecular Dynamics Docking to the Active Catalytic Site of SARS-CoV and SARS-CoV-2 Main Protease, Social Science Research Network(2020), Available

at: https://chemrxiv.org/articles/preprint/In_Silico_Molecular_Dynamics_Docking_of_Drugs to the_Inh ibitory Active Site of SARS-CoV2 Protease and Their Predicted Toxicology and ADME/12155523

27. A. Mishra, Y. Pathak, G. Choudhir, A. Kumar, S. K. Mishra, and V. Tripathi, Research Square(22 June, 2020)(2020), Available at: https://www.researchsquare.com/article/rs-22839/v2.pdf

28. S. T. Ngo, N. Quynh Anh Pham, L. Thi Le, D.-H. Pham, and V. V. Vu, Journal of Chemical Information and modeling, (epub date: June 12) (2020), https://doi.org/10.1021/acs.jcim.0c00491

29. M. Kateryna and V. S. Anna, ChemRXiv(epub date: 29 May 2020)(2020), Available at: https://chemrxiv.org/articles/preprint/Combined Use of Amentoflavone and Ledipasvir Could Int erfere with Binding of Spike Glycoprotein of SARS-CoV2 to ACE2 The Results of Molecular Docking Study/12377870

30. S. Megantara, J. Levita, S. Ibrahim, and B. P. Nguyen, Rasayan Journal of Chemistry, 14(2020), https://doi.org/10.31788/RJC.2021.1416070

31. S. S. Murthy, T. B. Narsaiah, Rasayan Journal of Chemistry, 4(2019), https://doi.org/10.31788/RJC.2019.1245475

32. M. Muchtaridi, D. Dermawan, M. Yusuf, Journal of Young Pharmacists, 10(3), 253(2018), https://doi.org/10.5530/jyp.2018.10.58

33. M. Muchtaridi, S. Megantara, D. Dermawan, M. Yusuf, Rasayan Journal of Chemistry, 12(2019), https://doi.org/10.31788/RJC.2019.1245391

[RJC-6172/2020] 